\title{
Optimizing the Amplifier Bandwidth for Pulse Reception.
}

\author{
Russell M. Kurtz, Senior Member, IEEE
}

\begin{abstract}
Detecting and recognizing pulses is a critical task, in fields as widely separated as telecommunications, lidar, and target illumination. In all cases, the signal-to-noise ratio (SNR) is a key parameter that can be used to determine both the potential rate of errors and the probability of correct detection. In this paper the relationship among pulse width, amplifier bandwidth, and SNR is determined through modeling four approximations to pulse shapes and four amplifier lowpass filter configurations. The analysis determined that, given a specific filter and pulse shape, the bandwidth that maximizes SNR is a constant divided by the pulse width. For example, if the pulse has a Gaussian shape and the amplifier incorporates a second-order Chebyshev lowpass filter, this constant is $\mathbf{0 . 3 3 8 9}$. Applying this, if the pulse width is $20 \mathrm{~ns}$ the maximum SNR comes for a filter bandwidth of $16.95 \mathrm{MHz}$, while if the pulse width is $50 \mu \mathrm{s}$ the SNR is maximized at a $6.778-\mathrm{kHz}$ bandwidth. Passing the signal through a filter also distorts the signal shape; the temporal shift and pulse lengthening are also determined. The calculated values are offered as inputs to a potential trade space that includes SNR, pulse distortion by the filter, and cost.
\end{abstract}

Keywords: Pulse reception, laser radar, tracking, target illumination, pulse amplification, optimum bandwidth.

\section{INTRODUCTION}

I nformation is passed as pulses of energy in many ways. These may be, for example, reflections from an item that is being illuminated by a laser [1], digital pulses encoded in microwave transmissions, or strings of electrical pulses sent along a communication line [2]. In most cases these pulses are converted into electricity so they can be amplified and interpreted. The amplifier will include some form of lowpass filtering system in frequency to reduce noise while amplifying the signal, since the signal-tonoise ratio (SNR) is a key indicator of how well that signal can be understood or even detected reliably [3].

The shorter the pulse width, the wider the amplifier bandwidth needs to be in order to receive the signal [4]. On the other hand, virtually all noise sources, including detector noise, thermal noise, photon noise, etc., are modeled well as white noise, whose magnitude is constant at all frequencies; as a result, the total noise value increases with filter bandwidth [5]. Increasing this bandwidth increases the signal amplitude, but with diminishing returns; increasing the bandwidth increases the noise at a constant rate. It is clear, then, that there is a point at which continuing to increase the filter bandwidth decreases the SNR.

In this paper the SNR effects of increasing the bandwidth are investigated, with the result that, over a wide variety of pulse shapes and amplifier types, the maximum value of the SNR comes when there is a simple inverse relationship between the pulse width, $\Delta t$, and the amplifier bandwidth, $\Delta v$. The analysis indicates that, for each specific lowpass filter configuration, the product $\Delta v \Delta t$ that maximizes SNR is a constant value, referred to as $\alpha_{p k}$. The modeling in this paper determines that value for four pulse shapes and four amplifier filters. We model each pulse as independent, rather than as part of a pulse stream, which is valid as long as the electronic manipulation of one pulse's signal takes place before the next pulse is received.
Another bandwidth consideration is that the temporal center and shape of the pulse are usually modified by the lowpass filter. Increasing the bandwidth far beyond the SNR peak reduces the pulse distortion. This is discussed in Part II, and used to provide information for a trade space that can help optimize lowpass filter bandwidth.

\section{Signals And Transforms}

Four pulse shapes were analyzed for this paper. Two are common approximations to pulse shapes, the rectangle (or $\Pi$ ) and the sinusoid pulse. The other two are more accurate representations of real-world pulses, the Gaussian and a shape referred to here as the Gamma pulse. The frequency-domain representations of the pulses are the magnitudes of their Laplace transforms.

The pulses, amplifiers, and responses are all studied in their electrical formats. If an optical pulse whose power is described as $P(t)$ is captured by a photodetector, the signal from the detector can then be defined in terms of current, $I(t)$, where the current at any time is directly proportional to the power reaching the detector at that time. The entire pulse, whose initial energy is $E_{0}$, produces a detector output whose total charge is $Q_{0}$. Direct electrical signals can also be analyzed in terms of the current as a function of time.

In this analysis, $t_{0}$ refers to the time of the peak of the pulse, and $\Delta t$ is the temporal width of the pulse measured as its full width at half maximum (FWHM).

The simplest approximation to a pulse is the rectangle, which is 0 outside the time in which the pulse is active and the maximum value within that time. Mathematically, this can be defined as

$$
I_{R}(t)=\frac{Q_{0}}{\Delta t} \Pi_{R}\left(\frac{t-t_{0}}{\Delta t}\right),
$$

where the modified $\Pi_{R}$ function is defined as

$$
\Pi_{R}(\xi) \equiv\left\{\begin{array}{cc}
0 & |\xi|>1 / 2 \\
1 / 2 & |\xi|=1 / 2 \\
1 & |\xi|<1 / 2
\end{array}\right.
$$

The raw $\Pi_{R}$ function has a peak value of 1 and a FWHM of 1. It is fully normalized:

$$
\int_{0}^{\infty} I_{R}(t) d t=Q_{0}
$$

as long as $t_{0} \geq \Delta t / 2$. All pulses will be normalized such that eq. (3) applies. The pulses are always described only in terms of $t>$ 0 , so that the Laplace transform provides an accurate description of the pulse in the frequency domain.

The design of a pulse forming network is often based on the attempt to produce a pulse whose shape is $1 / 2$ period of a sine function. This is difficult to achieve, but approximating a real-world pulse as a sinusoid pulse is usually more accurate than approximating it as a rectangle, at least for relatively short pulses. The sine pulse is defined mathematically as

$$
I_{S}(t)=\frac{\pi}{3} \frac{Q_{0}}{\Delta t} \cos \left(\frac{2 \pi}{3} \frac{t-t_{0}}{\Delta t}\right) \Pi_{R}\left(\frac{2}{3} \frac{t-t_{0}}{\Delta t}\right) .
$$

This pulse is also normalized to $Q_{0}$. 
Many short pulses can be approximated as Gaussian functions. For these functions, $t_{0}$ must be relatively large, so that there is essentially no charge in the pulse at times $t<0$. A normalized Gaussian pulse is

$$
I_{G}(t)=2 \sqrt{\frac{\ln (2)}{\pi}} \frac{Q_{0}}{\Delta t} \exp \left[-4 \ln (2)\left(\frac{t-t_{0}}{\Delta t}\right)^{2}\right] .
$$

In general, if $t_{0}>1.6 \Delta t$, eq. (5) is accurate to within $0.1 \%$, even when comparing the integral over only positive values of $t$ to that over all values of $t$. That accuracy is usually better than the accuracy of modeling the real-world pulse as a Gaussian function.

The function that seems to fit real-world pulses, such as $Q$-switched lasers and short telecom pulses, is based on $t \mathrm{e}^{-t}$. This fits the Gamma distribution with shape factor 2, so it is referred to in this analysis as a Gamma pulse. The normalized Gamma pulse has the mathematical description

$$
\begin{aligned}
I_{\Gamma}(t)= & \beta^{2} \frac{Q_{0}}{\Delta t^{2}}\left(t-t_{0}+\frac{\Delta t}{\beta}\right) \\
& \exp \left[-\frac{\beta}{\Delta t}\left(t-t_{0}+\frac{\Delta t}{\beta}\right)\right] U\left(t-t_{0}+\frac{\Delta t}{\beta}\right)
\end{aligned}
$$

where

$$
\beta \equiv W_{0}\left(-\frac{1}{2 e}\right)-W_{-1}\left(-\frac{1}{2 e}\right) \approx 2.446 .
$$

Here $W_{k}(z)$ is the $k^{\text {th }}$ branch of the Lambert $W$ function (or productlog) evaluated at $z$ and $U(z)$ is the unit step function. The unit step indicates that eq. is 0 for $t<t_{0}-\Delta t / \beta$.

Most of the analysis in this paper takes place in the frequency domain. Taking the Laplace transform [6] of a temporal function converts it to a function of frequency. Furthermore, the Laplace transform of $I(t)$ is $Q(s)$, where $s$ is the Laplace frequency. The full frequency representation of the function is the magnitude of $Q(s)$, evaluated at $s=i 2 \pi v$. Here $v$ is the frequency, measured in Hz. The mathematical description of the function's frequency description is

$$
Q(v)=|Q(s)|_{s=i 2 \pi v} \mid \text {. }
$$

As an example, consider the rectangle function of eq. (1). The Laplace transform of this is

$$
Q_{R}(s)=\int_{0}^{\infty} I_{R}(t) e^{-s t} d t=Q_{0} e^{-s t_{0}} \frac{\sinh (s \Delta t / 2)}{s \Delta t / 2} .
$$

Note that the integration over $t$ has converted $I_{R}(t)$ to $Q_{R}(s)$, resulting in the normalizing multiplier changing from $Q_{0} / \Delta t$ to $Q_{0}$. Evaluating eq. (9) at the frequency $s=i 2 \pi v$,

$$
\left.Q_{R}(s)\right|_{s=i 2 \pi v}=Q_{0} e^{-i 2 \pi t_{0} v} \operatorname{sinc}(\pi \Delta t v) .
$$

Taking the magnitude of eq. (10), we see

$$
Q_{R}(v)=\left|Q_{R}(s)\right|_{s=i 2 \pi v}\left|=Q_{0}\right| \operatorname{sinc}(\pi \Delta t v) \mid .
$$

The other Laplace transforms, and their magnitudes, are readily calculated as well. For the sinusoid pulse, we find

$$
Q_{S}(v)=Q_{0}\left|\frac{\cos (3 \pi \Delta t v / 2)}{1-(3 \Delta t v)^{2}}\right| .
$$

For the more realistic pulse models, the Gaussian pulse transforms to

$$
Q_{G}(v)=\frac{Q_{0}}{2} \exp \left[-\frac{(\pi \Delta t v)^{2}}{4 \ln (2)}\right] \operatorname{erfc}\left[\frac{-8 \ln (2)+i \pi \Delta t v}{2 \sqrt{\ln (2)}}\right],
$$

while the Gamma pulse transforms into a Lorentzian,

$$
Q_{\Gamma}(v)=Q_{0} \frac{\beta^{2}}{\beta^{2}+(2 \pi \Delta t v)^{2}} .
$$

The pulse functions, in both time (current normalized to $Q_{0} / \Delta t$ ) and frequency (charge normalized to $Q_{0}$ ), are shown in Fig. 1 .
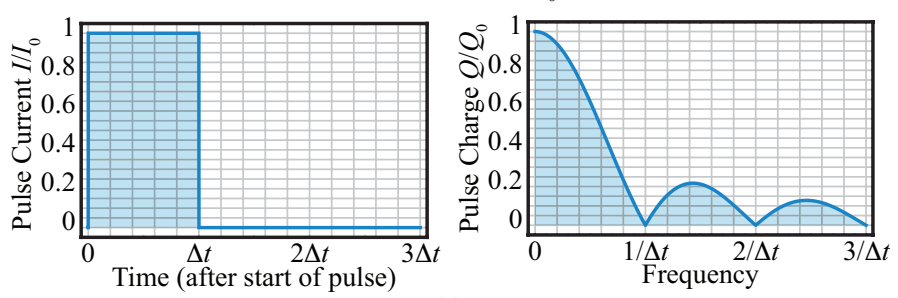

(a)
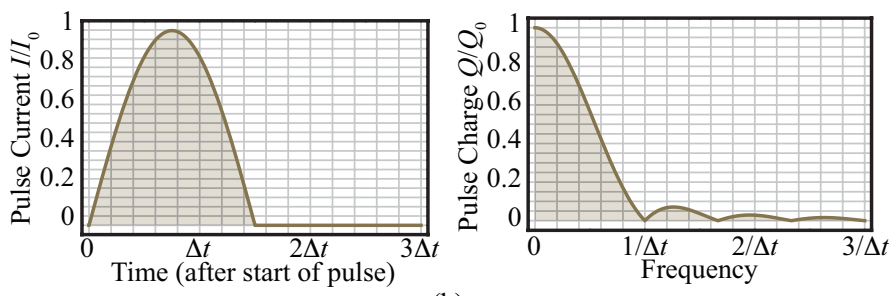

(b)
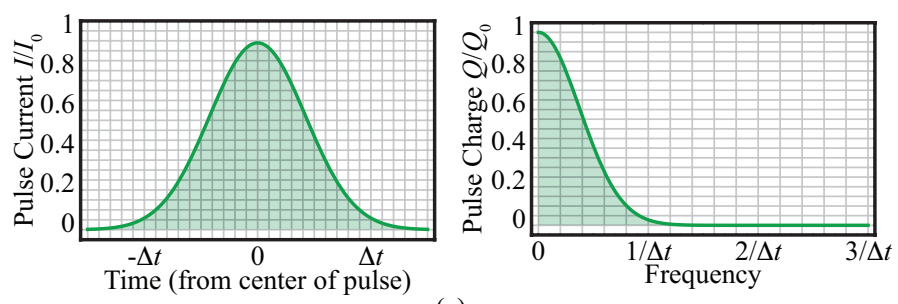

(c)
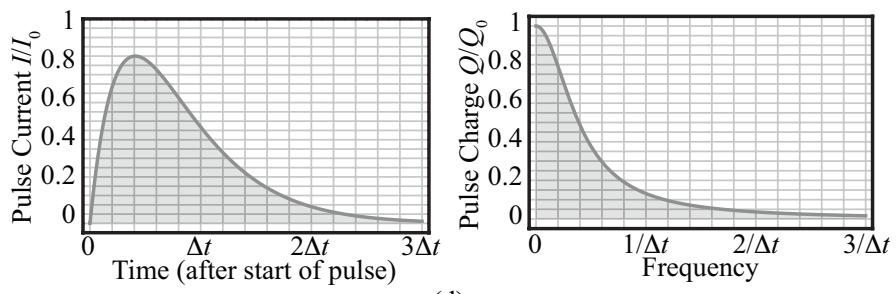

(d)

Fig. 1. The analyzed pulses in both time and frequency domains (a) rectangle, (b) sinusoid, (c) Gaussian, and (d) Gamma.

\section{Amplifiers AND Noise}

The amplifiers are modeled as incorporating lowpass filters. Three filter families are considered: Butterworth, which equalizes ripple in the passband and the stopband; Chebyshev (Type 1), which has the most rapid initial drop and minimized ripple in the stopband; and Bessel, which minimizes the signal distortion due to phase change. It was determined that optimized first-order Butterworth, Chebyshev, and Bessel filters all have the same magnitude, so while all three families are analyzed in second order, the fourth filter is just "first-order." It should be noted as well that the first-order filter can be the simplest electronic filter available, consisting of one resistor and one capacitor.

An amplifier is a filter incorporating gain. It is modeled here as having a gain $g(v)$, with units that do not vary with time or frequency. The filter portion of the amplifier is assumed to be purely lowpass, and it is characterized by the frequency at which its gain has fallen by a factor of $\sqrt{2}$, or $\sim 3 \mathrm{~dB}$. This frequency is designated $\Delta v$ and referred to as the bandwidth (or filter bandwidth).

An amplifier whose peak gain is $g_{0}$, using a first-order filter, 
has the mathematical description

$$
g_{1}(v)=\frac{g_{0}}{\sqrt{1+(v / \Delta v)^{2}}} .
$$

This is valid for all three filter families. A second-order $g_{0}$-gain Butterworth amplifier with a bandwidth of $\Delta v$ has an equally simple mathematical description,

$$
g_{B W 2}(v)=\frac{g_{0}}{\sqrt{1+(v / \Delta v)^{4}}} .
$$

An optimized second-order Chebyshev amplifier [7] with the same parameters is described by

$$
g_{C B 2}(v)=\frac{g_{0} / \sqrt{2}}{\sqrt{1-2(v / \Delta v)^{2}+2(v / \Delta v)^{4}}} .
$$

The equivalent optimized second-order Bessel amplifier has a somewhat similar description,

$$
g_{B S 2}(v)=\frac{g_{0}}{\sqrt{1+(v / \sqrt{\phi} \Delta v)^{2}+(v / \sqrt{\phi} \Delta v)^{4}}},
$$

where

$$
\phi \equiv \frac{1+\sqrt{5}}{2}
$$

is the golden ratio. The frequency responses of these four amplifiers are shown in Fig. 2.

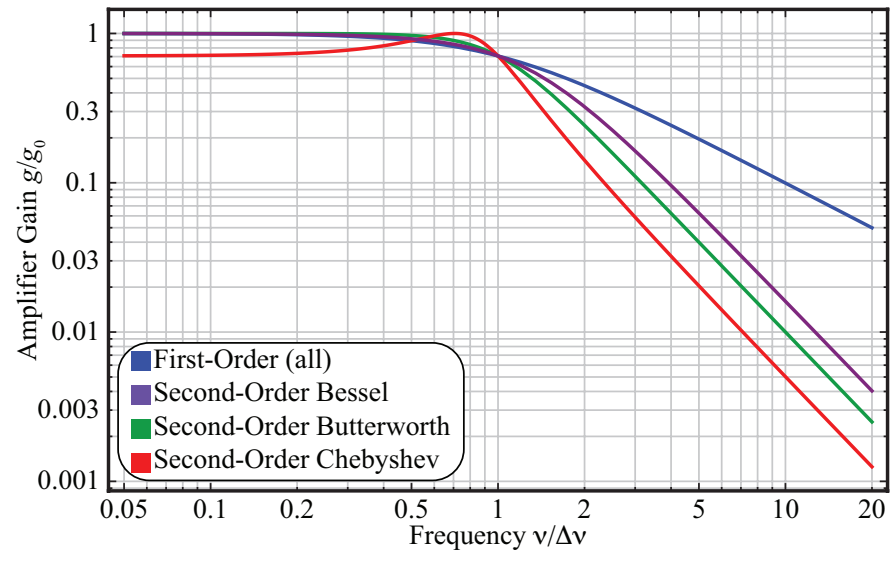

Fig. 2. Comparison of amplifier frequency response.

All the second-order filters have the same roll-off rate at high frequency, twice that of the first-order filter (in log-log units). Near $\Delta v$, however, the Chebyshev has the sharpest decline; it also suffers from a lower gain at low frequency. The Bessel has the longest transition from flat gain to roll-off, rivaling that of the first-order amplifier. These filtering shapes affect both the amplified signal and the total noise passed by the amplifier.

Electrical noise is normally analyzed as a random process. In this paper, two additional, standard assumptions are made: (1) the noise has a mean value of 0 , and (2) the amplitude of the noise is independent of frequency (it is white noise). Such noise sources are expressed in terms of a root-mean-squared (RMS) figure with units such as $\mathrm{A} / \sqrt{\mathrm{Hz}}$ or $\mathrm{W} / \sqrt{\mathrm{Hz}}$.

To determine the total noise anticipated at the output of an amplifier that incorporates a lowpass filter, the noise, multiplied by the amplifier characteristics, is averaged over all frequencies. Since the noise is a zero-mean random distribution, the noise variance is calculated; modeling the noise as a continuous distribution, this is the same as the mean-square noise:

$$
\sigma_{n}^{2}=\int_{0}^{\infty} i_{n}^{2}(v) g^{2}(v) d v=i_{\mathrm{RMS}}^{2} \int_{0}^{\infty} g^{2}(v) d v,
$$

where $i_{\mathrm{RMS}}$ is the RMS noise figure. The total RMS noise is the square root of this variance. As an example, consider a total input-referred noise figure of $i_{\text {RMS }} \mathrm{A} / \sqrt{\mathrm{Hz}}$ passed through an amplifier whose peak gain is $g_{0}$, incorporating a second-order Chebyshev lowpass filter whose bandwidth is $\Delta v$. The total RMS noise is then

$$
\begin{aligned}
\sigma_{C B 2} & =\sqrt{i_{\mathrm{RMS}}^{2} \frac{g_{0}^{2}}{2} \int_{0}^{\infty} \frac{d v}{1-2(\Delta v / v)^{2}+2(\Delta v / v)^{4}}}, \\
& =\left(\frac{1+\sqrt{2}}{32} \pi^{2}\right)^{1 / 4} g_{0} \sqrt{\Delta v} i_{\mathrm{RMS}}
\end{aligned},
$$

with the requirement that both $g_{0}$ and $i_{\text {RMS }}$ are real constants. The units of $\sigma_{n}$ are A (multiplied by the units of $g_{0}$, if any). Similar calculations result in total noise values for each type of filter as shown in Table 1.

Table 1. Total output noise based on amplifier filtering and input-referred noise figure.

\begin{tabular}{|c|c|c|}
\hline Filter & Total Noise & Numerical Value \\
\hline \hline First-Order & $\sqrt{\pi / 2} g_{0} \sqrt{\Delta v} i_{\mathrm{RMS}}$ & $1.253 g_{0} \sqrt{\Delta v} i_{\mathrm{RMS}}$ \\
\hline $\begin{array}{c}\text { Second-Order } \\
\text { Butterworth }\end{array}$ & $\left(\pi^{2} / 8\right)^{1 / 4} g_{0} \sqrt{\Delta v} i_{\mathrm{RMS}}$ & $1.054 g_{0} \sqrt{\Delta v} i_{\mathrm{RMS}}$ \\
\hline $\begin{array}{c}\text { Second-Order } \\
\text { Chebyshev }\end{array}$ & $\left(\frac{1+\sqrt{2}}{32} \pi^{2}\right)^{1 / 4} g_{0} \sqrt{\Delta v} i_{\mathrm{RMS}}$ & $0.9289 g_{0} \sqrt{\Delta v} i_{\mathrm{RMS}}$ \\
\hline $\begin{array}{c}\text { Second-Order } \\
\text { Bessel }\end{array}$ & $\left(\pi^{2} \phi / 12\right)^{1 / 4} g_{0} \sqrt{\Delta v} i_{\mathrm{RMS}}$ & $1.074 g_{0} \sqrt{\Delta v} i_{\mathrm{RMS}}$ \\
\hline
\end{tabular}

Faster amplifier roll-off, naturally, correlates with a lower total noise output. If it does not reduce the signal as much, it will also correlate with higher SNR.

\section{Maximizing the SNR}

This paper assumes that the maximum value of SNR occurs when the parameter defined here as $\alpha_{p k}$, which is the product of the amplifier bandwidth with the pulse width (FWHM), is a specific value independent of the bandwidth or pulse width selected. In other words,

$$
\mathrm{SNR}_{\text {max }}=f\left(\alpha_{p k}\right)=f(\Delta v \Delta t) \neq f(\Delta v, \Delta t),
$$

This assumption is justified by the numeric results presented in this section.

The value of $\Delta v \Delta t$ that maximizes SNR, $\alpha_{p k}$, will depend on the specific filter used in the amplifier and on the specific pulse shape, but the largest effect comes from the bandwidth-pulse width product. The specific amplifier gain, pulse noise figure, and signal strength do not affect the value of $\alpha_{p k}$ (although signal strength can contribute to the size of $i_{\mathrm{RMS}}$ through shot noise). In this section the SNR is calculated and maximized, and values of $\alpha_{p k}$ are calculated.

The noise, which is independent of frequency, pulse shape, etc., but dependent on bandwidth, was calculated in Section III. The signal is calculated as

$$
I_{t o t}=\int_{0}^{\infty} Q(v) g(v) d v,
$$

and has units of A multiplied by the units of $g_{0}$, if any. These are the same units as the noise, so the SNR is unitless.

Note that $Q(v)$ is usually dependent on $\Delta t$ (see Section II), just 
as $g(v)$ depends on $\Delta v$, so both $\Delta t$ and $\Delta v$ affect the value of eq. (23). The method used to find the $\alpha_{p k}$ (see Fig. 3) consists of six steps: (1) define the parameters (signal and amplifier type), (2) select a pulse width, (3) calculate the SNR for a range of bandwidths, (4) find the peak SNR and its related bandwidth for this pulse width, (5) repeat steps 3 and 4 for a range of pulse widths, and (6) list $\alpha \equiv \Delta v \Delta t$ for all pulse widths used, finding the peak value of $\Delta v$, $\Delta v_{p k}$, for each value of $\Delta t$. The value of $\alpha_{p k} \equiv \Delta v_{p k} \Delta t$ was found to be constant within the calculation error of the model.

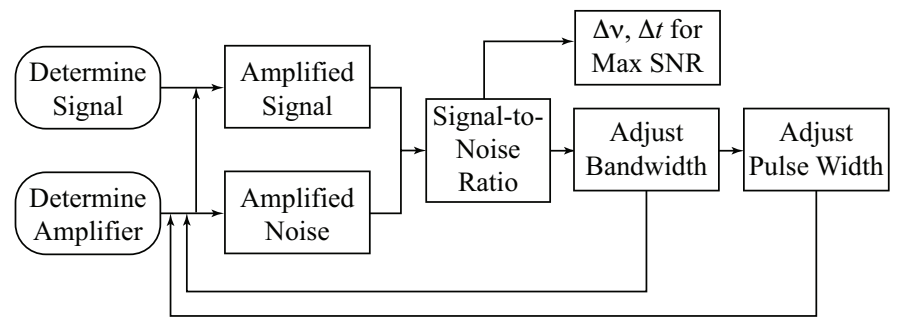

Fig. 3. Flowchart for calculating the value of $\alpha_{p k}$.

The signal calculation described by eq. (23) rarely has a closedform solution, so the integration was performed numerically in this analysis. In this case a Gaussian pulse passed through an amplifier incorporating a second-order Butterworth lowpass filter is used

as an example. The noise is $\left(\pi^{2} / 8\right)^{1 / 4} g_{0} \sqrt{\Delta v} i_{\text {RMS }}$ (see Section III), independent of the signal; the SNR for any combination of bandwidth and pulse width is calculated by first integrating the signal,

$$
\begin{aligned}
& I_{t o t G W 2}=\frac{Q_{0}}{2} g_{0} \\
& \int_{0}^{\infty} \frac{\exp \left[-\left(\frac{\pi \Delta t v}{2 \sqrt{\ln (2)}}\right)^{2}\right] \operatorname{erfc}\left[\frac{-8 \ln (2)+i \pi \Delta t v}{2 \sqrt{\ln (2)}}\right]}{\sqrt{1+\left(\frac{v}{\Delta v}\right)^{4}}} d v
\end{aligned}
$$

then dividing this calculation of $I_{t o t}$ by the total noise. The SNR is calculated for a range of values of $\Delta t$ from 0.05 to 5 ; for each value of $\Delta t$, the calculation is performed over a range of values of $\Delta v$ from 0.005 to 100 . The units are unimportant as long as those of $\Delta v$ are the inverse of those of $\Delta t$; for example, the units could be $\mathrm{kHz}$ and $\mathrm{ms}$, or $\mathrm{GHz}$ and $\mathrm{ns}$. The SNR calculations for this example are shown in Fig. 4.

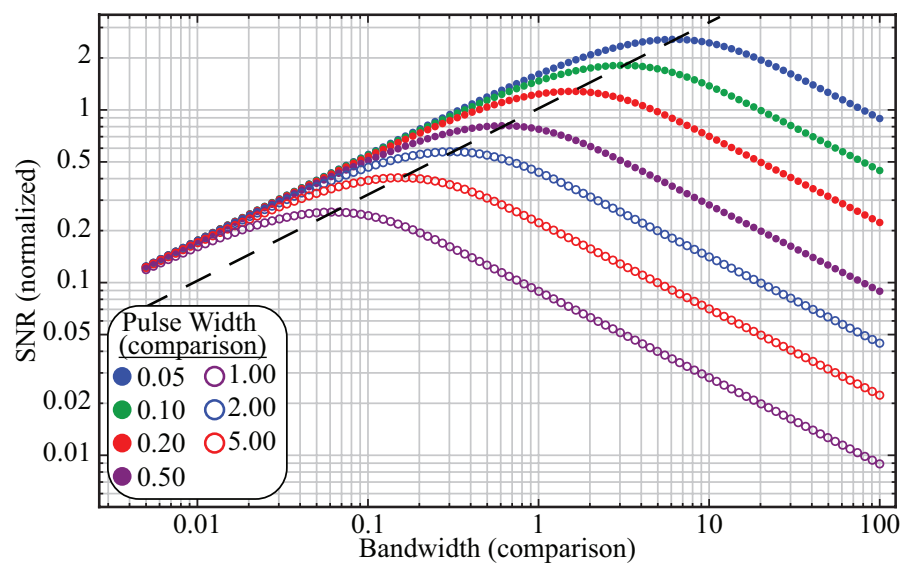

Fig. 4. SNR for a range of values of $\Delta v$ and $\Delta t$.

The dashed line indicates maximum SNR.

This figure shows SNR calculated for 200 values of $\Delta v$ at each of seven values of $\Delta t$. Bandwidth and pulse width have units listed as "comparison" indicating that the units are inverses of each other.
The SNR is normalized by dividing the calculated value by the magnitude of $Q_{0} / i_{\mathrm{RMS}}$, ignoring the units, which were eliminated in the SNR calculation.

The peak SNR value and bandwidth at which it occurs, for each modeled value of pulse width, are shown in Table 2. The relevant value of $\alpha$ is also listed. The peak SNR is directly proportional to $\sqrt{ } v v$, which indicates that $I_{t o t}$, as described by eq. (23), is also dependent on the $\Delta v \Delta t$ product rather than on pulse width or bandwidth independently.

Table 2. Calculations demonstrating that the peak SNR occurs at a point defined by $\alpha_{p k}$.

\begin{tabular}{|c|c|c|c|}
\hline Pulse Width $\Delta \boldsymbol{t}$ & Peak SNR & Bandwidth $\Delta v_{p k}$ & $\alpha_{p k} \equiv \Delta v_{p k} \Delta \boldsymbol{t}$ \\
\hline 0.05 & $2.561 Q_{0} / i_{\mathrm{RMS}}$ & 6.234 & 0.3117 \\
\hline 0.1 & $1.811 Q_{0} / i_{\mathrm{RMS}}$ & 3.117 & 0.3117 \\
\hline 0.2 & $1.281 Q_{0} / i_{\mathrm{RMS}}$ & 1.558 & 0.3117 \\
\hline 0.5 & $0.8099 Q_{0} i_{\mathrm{RMS}}$ & 0.6234 & 0.3117 \\
\hline 1 & $0.5727 Q_{0} / i_{\mathrm{RMS}}$ & 0.3117 & 0.3117 \\
\hline 2 & $0.4050 Q_{0} / i_{\mathrm{RMS}}$ & 0.1558 & 0.3117 \\
\hline 5 & $0.2561 Q_{0} / i_{\mathrm{RMS}}$ & 0.06234 & 0.3117 \\
\hline
\end{tabular}

The calculations described above were then repeated for all combinations of pulse shape and amplifier type. The results are shown in Fig. 5. The slope of each line is the inverse of the value of $\alpha_{\mathrm{pk}}$ for that combination of pulse shape and filter type (First $=$ first-order, $\mathrm{BW} 2$ = second-order Butterworth, $\mathrm{CB} 2$ = second-order Chebyshev, BS2 = second-order Bessel).

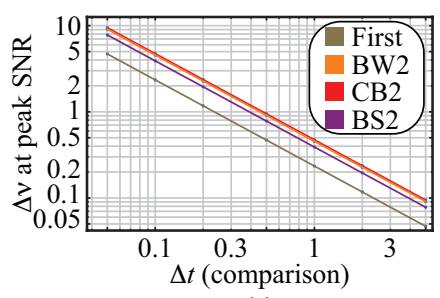

(a)

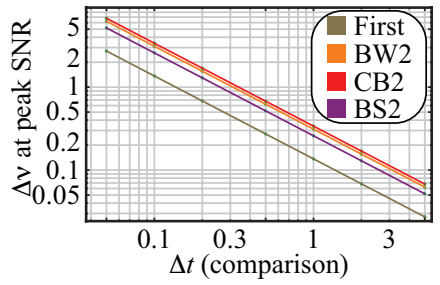

(c)

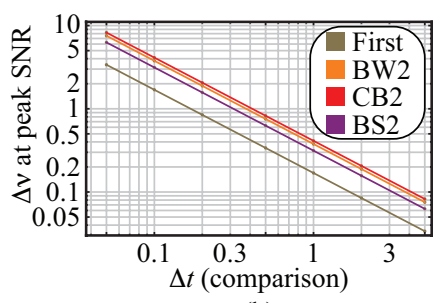

(b)

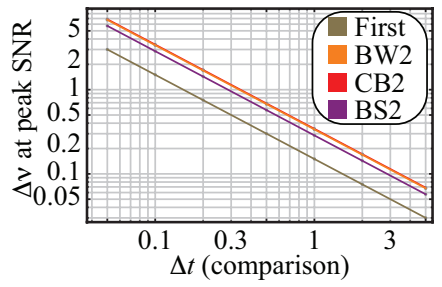

(d)
Fig. 5. Plots used to find $\alpha$ for four signals: (a) rectangle pulse, (b) sinusoid pulse, (c) Gaussian pulse, and (d) Gamma pulse.

Each pulse shape-amplifier type combination produces its own value of $\alpha_{p k}$ for the peak SNR. These are listed in Table 3.

Table 3. Values of $\alpha$ that maximize SNR $\left(\alpha_{p k}\right)$.

\begin{tabular}{|c|c|c|c|c|}
\hline Filter/Pulse & Rectangle & Sinusoid & Gaussian & Gamma \\
\hline First-Order & 0.2348 & 0.1691 & 0.1364 & 0.1502 \\
\hline Butterworth & 0.4543 & 0.3772 & 0.3117 & 0.3369 \\
\hline Chebyshev & 0.4739 & 0.4121 & 0.3389 & 0.3430 \\
\hline Bessel & 0.3902 & 0.3139 & 0.2589 & 0.2863 \\
\hline
\end{tabular}

The value of $\alpha_{p k}$ is lowest for amplifiers that use first-order filters. For amplifiers with second-order lowpass filters, the SNR is maximized at a value of $\alpha$ that is lowest for the Bessel and highest for the Chebyshev filter. This correlates inversely with the visible area under the curves in Fig. 2, where the Chebyshev is shown to have a more rapid roll-off for frequencies near $\Delta v$, the second-order 
Butterworth is next, the Bessel is the slowest onset of roll-off in the stopband for the three second-order filters analyzed, and the first-order filter has a slower roll-off at all frequencies above the passband cutoff.

The Gamma pulse is likely to be the most accurate representation of a real-world pulse (at least a short pulse), with the Gaussian pulse close to it. The pulse models with sharp discontinuitiesthe rectangle and sinusoid pulses - are generally less accurate. Furthermore, the sharp discontinuities lead to more significant charge values at high frequency. It is not surprising, then, that the Gamma and Gaussian pulses also have similar values of $\alpha_{p k}$, which are lower than those of the more idealized pulse models. The value of $\alpha_{p k}$ for a Gaussian pulse is consistently lower than that for the Gamma pulse, but the values are within $~ 10 \%$ of each other. The rectangle and sinusoid pulse produce $\alpha_{p k}$ values that are $20 \%-50 \%$ higher.

\section{Pulse Distortion}

This analysis, so far, has concentrated solely on maximizing SNR. It is necessary to also consider such factors as the cost and gain-bandwidth product (GBW) of the amplifier, the signal distortion induced by the filter, and the change in the determination of the pulse peak. The last point, the pulse peak offset, is modeled as being caused solely by the filter; any imperfections in the processing electronics have been ignored.

The cost and GBW of the amplifier are closely related [8]. First, the higher the GBW, in general, the higher the cost. In addition, larger bandwidths lead to more noise, even with components whose noise figures are related only to the GBW and not to the bandwidth alone. Price alone tends to recommend the lowest possible value of $\alpha$-although at values below the peak SNR, the trade-off becomes price vs. signal reliability. Thus, if the only consideration is whether or not the pulse can be detected (or how far away the pulse can be detected), the value of $\alpha$ that maximizes SNR optimizes the amplifier design.

If the purpose, however, requires precise temporal location or pulse shape recognition, other considerations can be important. If the time of a specific pulse (rather than, for example, the time between pulses) is critical, the peak offset is important. This can occur in lidar, since the time of flight of the pulse is related to the target's distance. Peak offset can also be an essential parameter when correlating the timing of separate events. Pulse shape can also be important when selecting a specific pulse out of a group.

These effects are shown in Fig. 6. Pulses were modeled as passing through amplifiers incorporating the modeled filters filters. In this model, the peak value of the input pulse is normalized to 1.0 , it is centered at $t_{0}$, and its FWHM pulse width is $\Delta t$. The output of the amplifier is calculated for five bandwidths: $0.5,1.0$, 1.5, 2.0, and 2.5 times the bandwidth that produces the peak SNR value (defined as $\Delta v_{p k}=\alpha_{p k} / \Delta t$ ), and the peak of each output is also scaled to 1.0. The original pulse and the half-maximum line are added for reference. These plots demonstrate both the offset of the signal peak and the distortion of the pulse shape.

It is clear that passing the pulse through a low-pass filter has altered the shape, sometimes dramatically, and has also affected the pulse width. The greater the bandwidth, the less effect on the pulse width, although Fig. 6(a) shows that the shape distortion can be significant even at high bandwidths.

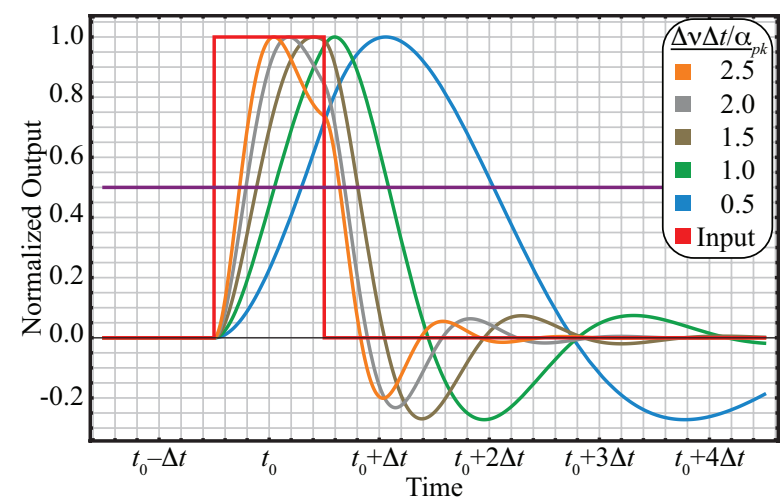

(a)

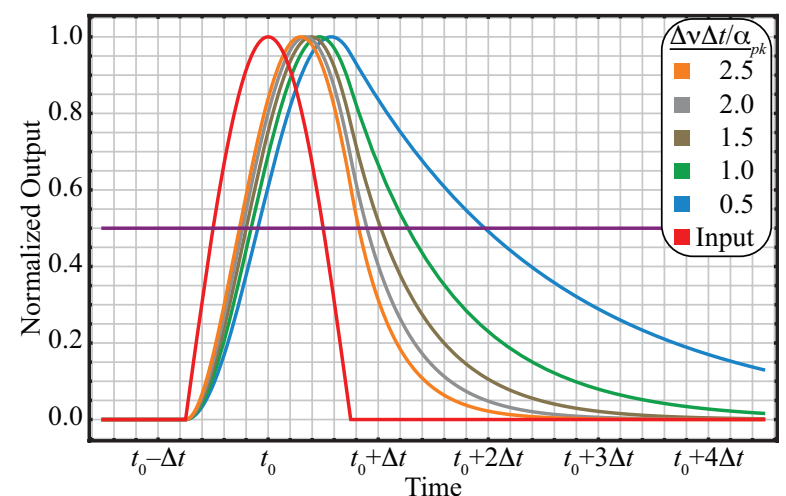

(b)

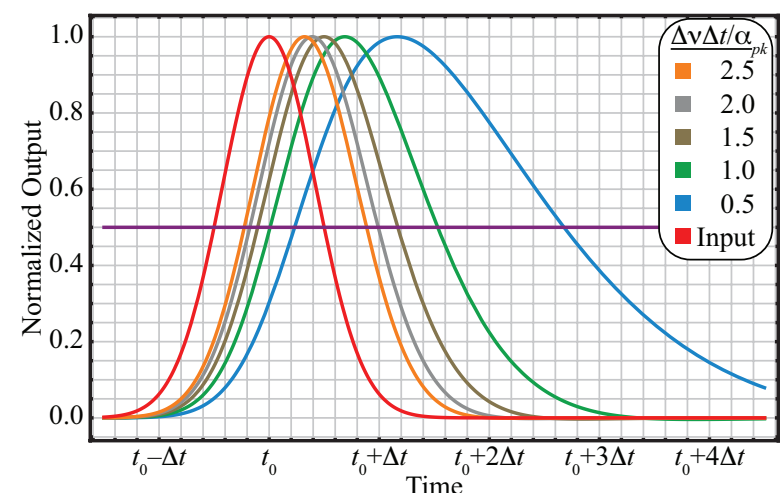

(c)

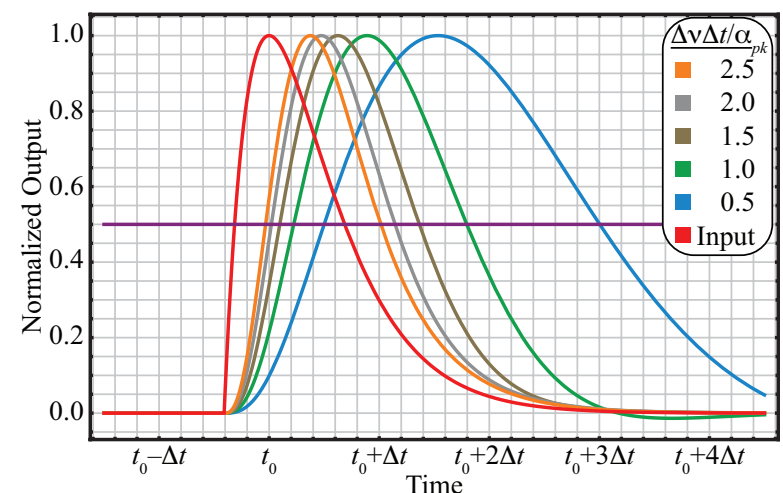

(d)

Fig. 6. Signal offset and distortion through amplifiers: (a) rectangular pulse with Chebyshev filter, (b) sinusoidal pulse with first-order filter, (c) Gaussian

pulse with Bessel filter, and (d) Gamma pulse with Butterworth filter.

Just as it is useful to calculate the SNR-optimized amplifier bandwidth for a given pulse width and shape, the shift of the peak value of the amplified pulse and its increase in FWHM are important. these can be modeled, resulting in an equation to predict these values. The model used in this paper was employed to determine 
the time of the peak and the pulse FWHM for 35 filter bandwidths from $\Delta v \Delta t / \alpha_{p k}=0.5-2.5$ - that is, from 0.5-2.5 $\times$ the bandwidth that produces the peak value of SNR. This range was assumed to be a reasonable range of bandwidths. A simple function, based on $a /$ $n^{b}$, was fit to the time shift of the peak, $t_{s h i f t} \equiv t_{p k}-t_{0}$; the FWHM ratio, $\delta t \equiv \Delta t_{\text {out }} / \Delta t$, was fit to $a / n^{\mathrm{b}}+c$. Data generated by the model showed that, in these fits, the parameters $a$ and $c$ scale linearly with $\Delta t$, while $b$ is independent. A much wider bandwidth range would require a more complicated fitting formula; even over this narrow range, the accuracy of the approximation is significantly less for a rectangular pulse than the other three pulse shapes modeled (Fig. 7). Interestingly, when using the Butterworth or Chebyshev filters, the modeled data for $\delta t$ drops below 1.0 for bandwidths greater than $1.5 \times$ the SNR-optimized value. These filters are slightly oscillatory with frequency and the modeled results come back to the value of 1.0 for near-infinite filter bandwidths.

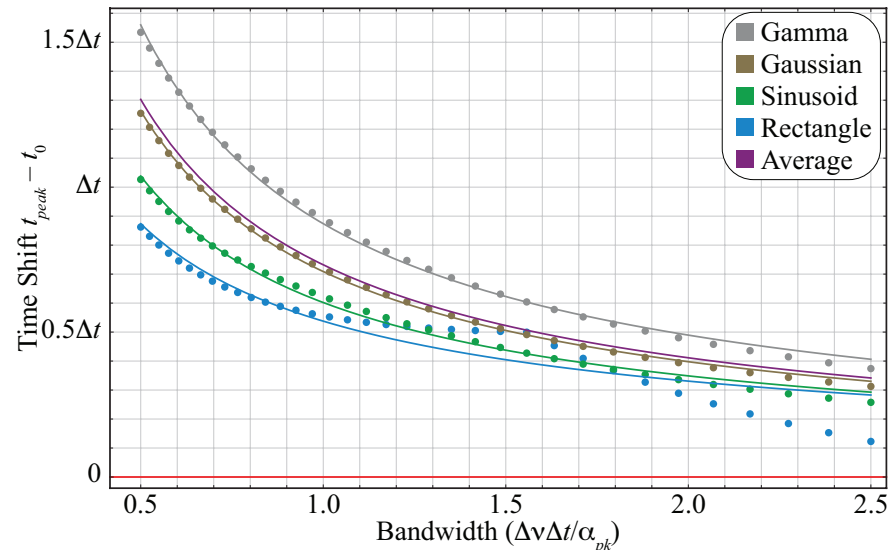

(a)

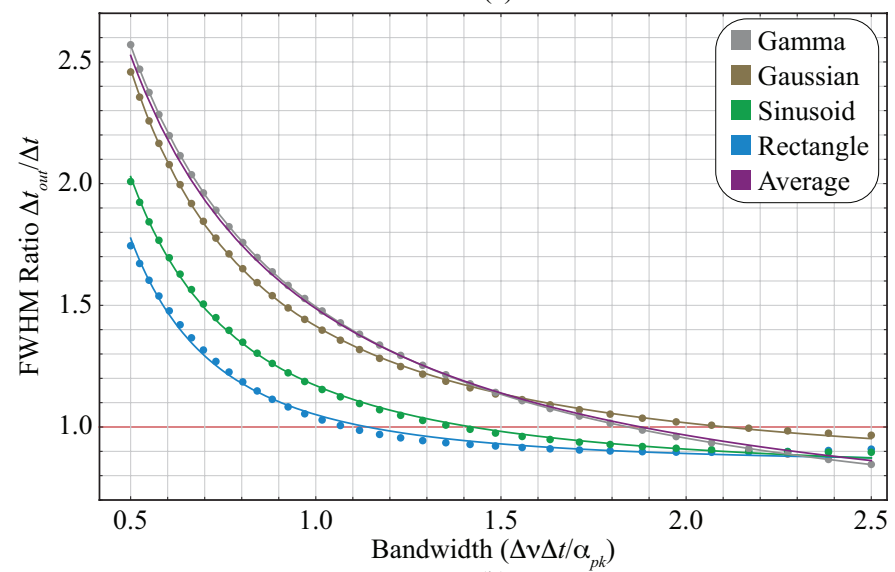

(b)

Fig. 7. Fitting curves to (a) the temporal location of the peak of the signal after passing through a second-order Butterworth low-pass filter and (b) the FWHM pulse width of the signal after passing through a second-order Chebyshev lowpass filter.

The curve-fitting (at least, over this narrow bandwidth region) is excellent, with $R^{2}$ goodness-of-fit values of $99.99 \%$ and better for all of them. In addition, a weighted average value was calculated, as a general rule of thumb approach. This average placed greater emphasis on the modeled Gaussian and Gamma pulses, rather than the Rectangle and Sine pulses. In addition, the curves that more closely fit the data were weighted more strongly than the others. The result closely approximates the curve for the Gaussian pulse when predicting the peak time shift, and closely approximates the curve for the Gamma pulse when estimating the pulse width.

The time shift is modeled as

$$
t_{\text {shift }} \equiv t_{p k}-t_{0} \approx \frac{a}{n^{b}},
$$

where $n \equiv \Delta v \Delta t / \alpha_{\mathrm{pk}}$ is the ratio of the filter bandwidth to the bandwidth that optimizes the SNR for a pulse width of $\Delta t$. The parameters $a$ and $b$ that optimize the curve fit for each combination of filter and pulse shape are listed in Table 4.

Table 4. Curve fit parameters that optimize the fit to $t_{\text {shift }}$.

\begin{tabular}{|c|c|c|c|c|}
\hline Filter: & \multicolumn{2}{|c|}{ First-Order } & \multicolumn{2}{c|}{ Butterworth } \\
\hline Pulse Shape & $a / \Delta t$ & $b$ & $a / \Delta t$ & $b$ \\
\hline Rectangle & 0.5 & 0 & 0.5376 & 0.7007 \\
\hline Sinusoid & 0.4540 & 0.3896 & 0.7087 & 0.7867 \\
\hline Gaussian & 0.4887 & 0.4275 & 0.7087 & 0.8335 \\
\hline Gamma & 0.6956 & 0.5019 & 0.8738 & 0.8360 \\
\hline Average & 0.5334 & 0.4620 & 0.7320 & 0.8317 \\
\hline Filter: & \multicolumn{2}{|c|}{ Chebyshev } & \multicolumn{2}{c|}{ Bessel } \\
\hline Rectangle & 0.5446 & 1.026 & 0.5660 & 0.3810 \\
\hline Sinusoid & 0.6386 & 0.9605 & 0.5854 & 0.7136 \\
\hline Gaussian & 0.7753 & 0.9575 & 0.6815 & 0.7779 \\
\hline Gamma & 1.003 & 0.9041 & 0.8335 & 0.7933 \\
\hline Average & 0.8392 & 0.9330 & 0.7013 & 0.7788 \\
\hline
\end{tabular}

The fit to the pulse width ratio,

$$
\delta t \equiv \frac{\Delta t_{\text {out }}}{\Delta t} \approx \frac{a}{n^{b}}+c,
$$

is somewhat more complex, having three parameters $(a, b$, and $c)$. These parameters are listed in Table 5.

Table 5. Curve parameters that optimize the fit to $\delta t$.

\begin{tabular}{|c|c|c|c|c|c|c|}
\hline Filter: & \multicolumn{3}{|c|}{ First-Order } & \multicolumn{3}{c|}{ Butterworth } \\
\hline Pulse Shape & $a / \Delta t$ & $b$ & $c / \Delta t$ & $a / \Delta t$ & $b$ & $c / \Delta t$ \\
\hline Rectangle & 0.1835 & 1.662 & 0.9579 & 0.1416 & 2.414 & 0.9473 \\
\hline Sinusoid & 0.5232 & 1.143 & 0.8906 & 0.3251 & 1.771 & 0.9066 \\
\hline Gaussian & 0.8040 & 1.038 & 0.9453 & 0.5845 & 1.408 & 0.9002 \\
\hline Gamma & 1.234 & 0.7878 & 0.8066 & 0.8552 & 1.066 & 0.7161 \\
\hline Average & 0.8393 & 0.9660 & 0.9309 & 0.6586 & 1.186 & 0.8385 \\
\hline Filter: & \multicolumn{3}{|c|}{ Chebyshev } & \multicolumn{4}{c|}{ Bessel } \\
\hline Rectangle & 0.2048 & 2.183 & 0.8460 & 0.1345 & 2.361 & 0.9650 \\
\hline Sinusoid & 0.3753 & 1.717 & 0.3753 & 0.3279 & 1.713 & 0.9325 \\
\hline Gaussian & 0.6381 & 1.408 & 0.7766 & 0.5823 & 1.376 & 0.9404 \\
\hline Gamma & 1.086 & 0.9920 & 0.4079 & 0.8181 & 1.055 & 0.8148 \\
\hline Average & 1.041 & 0.9996 & 0.4456 & 0.6465 & 1.182 & 0.8993 \\
\hline
\end{tabular}

As can be seen from Fig. 7, even just using the average to calculate $t_{\text {shift }}$ and $\delta t$ will provide a reasonable prediction, especially since it is not always easy to determine what the actual pulse shape will be. It can be important, then, to determine how much pulse distortion is acceptable. One possible guide to this is the derivative of the predictive curve. We know that at very low bandwidths, the distortion is large, and any increase in bandwidth will result in a significant reduction of this distortion. At large bandwidths, where the distortion is small, increasing the bandwidth will only increase the cost and complexity of the amplifier while decreasing SNR, with little reduction in $t_{\text {shift }}$ or $\delta t$. As a possible guide to this, Fig. 8 shows the derivatives of each fit with respect to the normalized bandwidth $\mathrm{n} \equiv \Delta v \Delta t / \alpha_{p k}$, where $\Delta v$ is the actual filter bandwidth, $\Delta t$ is the actual pulse width, and $\alpha_{p k}$ is the SNR optimization parameter caclulated in Section IV. 


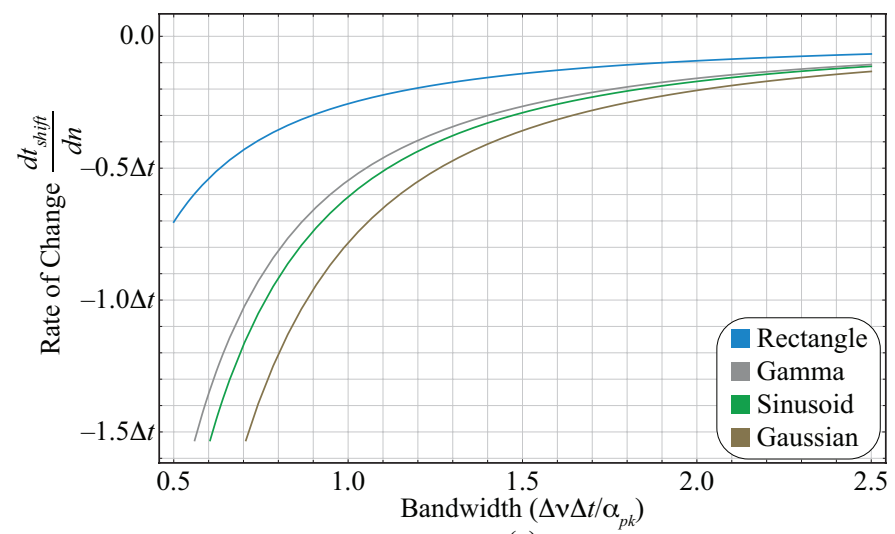

(a)

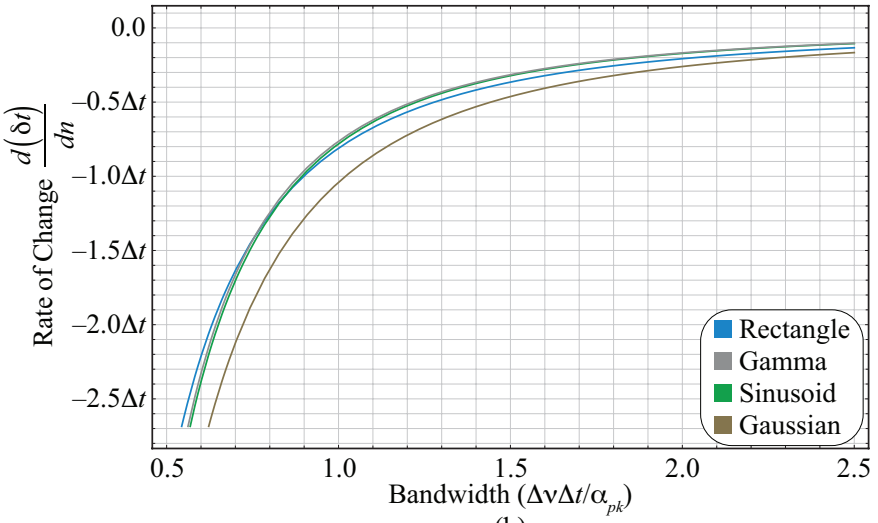

(b)

Fig. 8. Derivatives of distortion factors (a) peak time shift and (b) pulse width, with respect to bandwidth.

Note that, since $n \equiv \Delta v \Delta t / \alpha_{p k}$,

$$
\frac{d}{d n} f(n)=\frac{\alpha_{p k}^{p k}}{\Delta t} \frac{d}{d(\Delta v)} f(\Delta v) .
$$

In other words, the rate of change of both $t_{\text {shift }}$ and $\delta t$ with respect to the normalized bandwidth $n$ is a linear multiple of the rate of change of that distortion with respect to the bandwidth. For example, the values at which the rate of change of the peak equals $0.5 \Delta t$ are $0.6321 \Delta t$ for the first-order filter average, $1.113 \Delta t$ for the second-order Butterworth, 1.261 $\Delta t$ for the Chebyshev, and 0.7911 $\Delta t$ for the Bessel filter. To reach the same rate of change for $\delta t$, the required bandwidths are $1.279 \Delta t, 1.226 \Delta t, 1.443 \Delta t$, and $1.214 \Delta t$ for the first-order, Butterworth, Chebyshev, and Bessel filters, respectively. To reach a rate of change of $0.2 \Delta t$, the Chebyshev filter model requires a bandwidth of $2.281 \Delta t$, significantly higher.

At a bandwidth of $1.113 \Delta t$, which corresponds to $d t_{\text {shift }} /$ $d n=0.5 \Delta t$ for the Butterworth filter, the calculated value of $t_{\text {shift }}$ with this filter is $0.6694 \Delta t$, while $\delta t=1.418$. At a bandwidth of $1.443 \Delta t$ and a Chebyshev filter, the value of $d(\delta t) / d n$ is $0.5 \Delta t$, the calculated value of $\delta t$ is 1.167 , and $t_{\text {shift }}=0.596 \Delta t$. The distortions are significantly reduced by $1.2-1.5 \times$ the bandwidth that maximizes the SNR, while calculations such as those plotted in Fig. 4 indicate that the SNR itself drops $<10 \%$ when the filter bandwidth is doubled (or halved). Reducing pulse distortion may recommend a bandwidth increase of between $20 \%$ and $100 \%$ as a starting point in the trade space that can optimize the required filtered pulse parameters, depending on the specific application.

\section{Conclusions}

It is proposed that, given the temporal width, $\Delta t$, of an electrical pulse, the amplifier bandwidth, $\Delta v$, that maximizes the SNR of the received pulse is simply related to the pulse width through the equation

$$
\Delta v_{p k}=\frac{\alpha_{p k}}{\Delta t}
$$

where $\alpha_{p k}$ is a dimensionless constant that depends, in general, on the shape of the pulse and the type of frequency filtration used by the amplifier. This is demonstrated by a model that uses four pulse shapes and four lowpass filters. The value of $\alpha_{p k}$ is shown to depend more strongly on $\Delta t$ than on the details of the pulse shape, especially for realistic shapes, and to depend much more strongly on the order of the lowpass filter than on its implementation.

This analysis specified $\Delta v$ (traditionally) as the 3-dB bandwidth of the amplifier's lowpass filter, defining it through the equation $g(\Delta v)=g_{0} / \sqrt{2}$ (see Section III). (It should be noted that, for the Chebyshev lowpass filter, this definition requires $v$ $>0$, since at $v=0$ the gain is the same as at $v=\Delta v$.) In a similar fashion, $\Delta t$ was defined as the FWHM pulse width in time. Using these definitions, there is not a closed-form correlation between specific filter parameters and the value of $\alpha_{p k}$. There is, however, a qualitative correlation between the integrated filter magnitude (or the integrated squared filter magnitude, since the integral of the first-order filter magnitude is infinite) and the inverse of $\alpha_{p k}$. The integral of the first-order filter is largest, followed by that of the second-order Bessel, then the second-order Butterworth, while the integral of the second-order Chebyshev is smallest. This is also true for the integrated squared values, as indicated by the noise calculations listed in Table 1 . Table 3 , listing the values of $\alpha_{p k}$ for the combination of amplifiers and signals modeled, indicates that the value of $\alpha_{p k}$ is lowest for the first-order filter, followed (in order) by the Bessel, Butterworth, and Chebyshev second-order filters - the opposite of the order of the integrated filter magnitudes.

Finally, this analysis mentions the GBW and the cost, and studies the pulse distortion caused by the filter in terms of peak time shift and pulse width increase. These are calculated separately and parameterized by the original pulse length $\Delta t$. Both distortions can be estimated, together with their sensitivity to increasing the filter bandwidth. This helps provide the basis for a trade space between pulse quality and amplified pulse SNR.

\section{REFERENCES}

1. Liedahl, D.A.; Rubenchik, A.; Libby, S.B., et al. "Pulsed Laser Interactions with Space Debris: Target Shape Effects.” Adv Space Res. 52(5): 895-915; 2013 Sep 1. doi:https://doi.org/10.1016/j.asr.2013.05.019.

2. Proakis, J.G.; Salehi, M. Digital Communications. Fifth Ed. New York: McGraw Hill; 2008. 1150 p.

3. Spieler, H. "Electronic Noise." ICFA Instrumentation School. Istanbul, Turkey, 2002. p. 1-35.

4. Kido, K.i. Digital Fourier Analysis: Fundamentals. New York, NY: Springer New York; 2015. doi:10.1007/978-1-4614-9260-3.

5. Kurtz, R.M.; Forrester, T.C. "Optimizing Reception Bandwidth of a Pulsed Signal.” In: Molebny, V., editor. Laser Radar Technologies and Applications XXV. Online Only, California: SPIE; 11410, 2020. p. 114100B. doi:10.1117/12.2558339.

6. Bryant, M.D. "Laplace Transforms \& Transfer Functions." Course Notes. University of Texas at Austin (Mechanical Engineering), ME344: Dynamic Systems and Controls. Mar 25, 2008.

7. Wolfram Research, Inc. Analog Filter Design. Champaign, Illinois: Mathemtatica ${ }^{\circledR}$ Documentation; [modified 2016]. Available from: https://reference.wolfram.com/ language/tutorial/AnalogFilterDesign.html.

8. Texas Instruments. Operational Amplifiers (Op Amps). [modified 2021]. Available from: https://www. ti.com/amplifier-circuit/op-amps/products.html. 